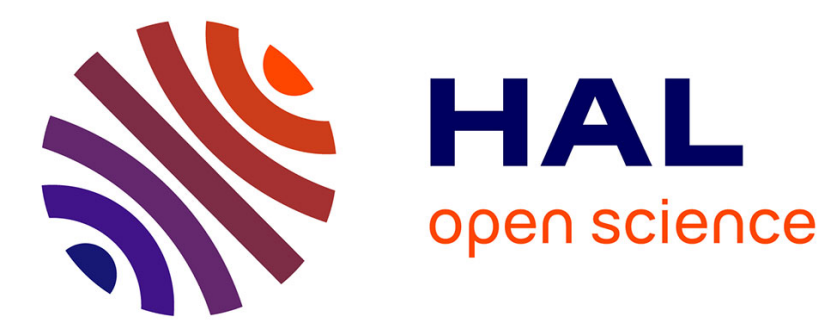

\title{
Statistical Model Checking for SystemC Models
}

van Chan Ngo, Axel Legay, Jean Quilbeuf

\section{To cite this version:}

van Chan Ngo, Axel Legay, Jean Quilbeuf. Statistical Model Checking for SystemC Models. High Assurance Systems Engineering Symposium, Jan 2016, Orlando, Florida, United States. hal-01238162

\section{HAL Id: hal-01238162 \\ https://hal.inria.fr/hal-01238162}

Submitted on 4 Dec 2015

HAL is a multi-disciplinary open access archive for the deposit and dissemination of scientific research documents, whether they are published or not. The documents may come from teaching and research institutions in France or abroad, or from public or private research centers.
L'archive ouverte pluridisciplinaire HAL, est destinée au dépôt et à la diffusion de documents scientifiques de niveau recherche, publiés ou non, émanant des établissements d'enseignement et de recherche français ou étrangers, des laboratoires publics ou privés. 


\title{
Statistical Model Checking for SystemC Models
}

\author{
Van Chan Ngo Axel Legay Jean Quilbeuf \\ INRIA, Campus Beaulieu, 35042 Rennes, France \\ Email: \{chan.ngo, axel.legay, jean.quilbeuf\}@inria.fr
}

\begin{abstract}
Transaction-level modeling with SystemC has been very successful in describing the behavior of embedded systems by providing high-level executable models, in which many of them have an inherent probabilistic behavior, i.e., random data, unreliable components. It is crucial to evaluate the quantitative and qualitative analysis of the probability of the system properties. Such analysis can be conducted by constructing a formal model of the system and using probabilistic model checking. However, this method is infeasible for large and complex systems due to the state space explosion. In this paper, we demonstrate the successful use of statistical model checking to carry out such analysis directly from large SystemC models and allows designers to express a wide range of useful properties.
\end{abstract}

Keywords-Runtime Verification, Probabilistic Assertion, Statistical Model Checking, Program Verification, SystemC

\section{INTRODUCTION}

Transaction-level modeling (TLM) with SystemC has been become increasingly prominent in describing the behavior of embedded systems [3], i.e., System-on-Chips (SoCs). It allows complex electronic components and software control units to be combined into a single model, enabling simulation of the whole system at once. In many cases, models include probabilistic characteristics, i.e, random data, reliability of the system's components. It is crucial to evaluate the quantitive and qualitative analysis of the probability of the system's properties. For instance, the reliability and availability of an embedded control system [14] that contains an input processor connected to groups of sensors, an output processor, connected to groups of actuators, and a main processor, that communicates with the I/O processors through a bus, can be modeled by a continuous-time Markov chain (CTMC) [25]. CTMC is a special case of a discrete-state stochastic process in which the probability distribution of the next state depends only on the current state. The analysis quantifies the probability or rate of all safety-related faults: How likely the system is available to meet a demand for service? What is the probability that the system repairs itself after a failure (e.g., that the system conforms to the existent and prominent standards such as the Safety Integrity Levels (SILs))?

In order to conduct such analysis, a general approach is modeling and analyzing a probabilistic model of the system (i.e, Markov chains, stochastic processes), in which the algorithm for computing the measures of properties depends on the class of systems being considered and the logic used for specifying the properties. Many algorithms with the corresponding mature tools are based on model checking techniques that compute probability by a numerical approach [4], [21], [9]. For a variety of probabilistic systems, the most popular modeling formalism is Markov chain or Markov decision processes, for which Probabilistic Model Checking (PMC) tools such as PRISM [10] and MRMC [13] can be used. PMC is widely used and has been successfully applied to the verification of a range of timed and probabilistic systems. One of the main challenges is the complexity of the algorithms in terms of execution time and memory space due to the size of the state space that tends to grow exponentially, also known as the state space explosion. As a result, the analysis is infeasible. In addition, these tools cannot work directly with the SystemC source code, meaning that a formal model of SystemC model needs to be provided.

An alternative way to evaluate these systems is Statistical Model Checking (SMC), a simulation-based approach. Simulation-based approaches produce an approximation of the value to be evaluated, based on a finite set of system's executions. Clearly, comparing to the numerical approach, a simulation-based solution does not provide an exact answer. However, users can tune the statistical parameters such as the confidence interval and the confidence, according to the requirements. Simulation-based approaches do not construct all the reachable states of the model-under-verification (MUV), thus they require far less execution time and memory space than numerical approaches. For some real-life systems, they are the only one option [28] and have shown the advantages over other methods such as PMC [9], [12].

Our overall framework weaves the idea of statistical model checking to yield qualitative and quantitative analysis for probability of a temporal property for SystemC models. The paper makes the following contributions: (i) we propose a framework to verify bounded temporal properties for SystemC models with both timed and probabilistic characteristics. The framework contains two main components: a monitor that observes a set of execution traces of the MUV and a statistical model checker implementing a set of hypothesis testing algorithms. We use techniques similar to the one proposed by Tabakov et al. [23] to automatically generate the monitor. The statistical model checker is implemented as a plugin of the checker Plasma Lab [2], in which the properties to be verified are expressed in Bounded Linear Temporal Logic (BLTL); (ii) we present a method that allows users to expose a rich set of usercode primitives in form of atomic propositions in BLTL. These propositions help users exposing the state of the SystemC simulation kernel and the full state of the SystemC source code model. In addition, users can define their own fine-grained time resolution that is used to reason on the semantics of the 
logic expressing the properties rather the boundary of clock cycles in the SystemC simulation; and (iii) we demonstrate our approach through a running example, in which we showcase how our SMC-based verification framework works. We also illustrate the performance of the framework through some experiments.

\section{BACKGROUND}

\section{A. SystemC and the Simulation Kernel}

SystemC ${ }^{1}$ is a $\mathrm{C}++$ library [6] providing primitives for modeling hardware and software systems at the level of transactions. Every SystemC model can be compiled with a standard $\mathrm{C}++$ compiler to produce an executable program called executable specification. This specification is used to simulate the system behavior with the provided event-driven simulator. A SystemC model is a hierarchical composition of modules (sc_module). Modules are building blocks of SystemC design, they are like modules in Verilog [24], classes in $\mathrm{C}++$. A module consists of an interface for communicating with other modules and a set of processes running concurrently to describe the functionality of the module. An interface contains ports (sc_port), they are similar to the hardware pins. Modules are interconnected using either primitive channels (i.e., the signals, sc_signal) or hierarchical channels via their ports. Channels are data containers that generate events in the simulation kernel whenever the contained data changes.

Processes are not hierarchical, so no process can call another process directly. A process is either a thread or a method. A thread process (sc_thread) can suspend its execution by calling the library statement wait or any of its variants. When the execution is resumed, it will continue from that point. Threads run only once during the execution of the program and are not expected to terminate. On the other hand, a method process (sc_method) cannot suspend its execution by calling wait and is expected to terminate. Thus, it only returns the control to the kernel when reaching the end of its body.

An event is an instance of the SystemC event class (sc_event) whose occurrence triggers or resumes the execution of a process. All processes which are suspended by waiting for an event are resumed when this event occurs, we say that the event is notified. A module's process can be sensitive to a list of events. For example, a process may suspend itself and wait for a value change of a specific signal. Then, only this event occurrence can resume the execution of the process. In general, a process can wait for an event, a combination of events, or for an amount of time to be resumed. In SystemC, integer values are used as discrete time model. The smallest quantum of time that can be represented is called time resolution meaning that any time value smaller than the time resolution will be rounded off. The available time resolutions are femtosecond, picosecond, nanosecond, microsecond, millisecond, and second. SystemC provides functions to set time resolution and declare a time object. The SystemC simulator is an event-driven simulation [1], [19]. It establishes a hierarchical network of

\footnotetext{
${ }^{1}$ IEEE Standard 1666-2005
}

finite number of parallel communicating processes which run under the supervision of the distinguished simulation kernel process. Only one process is dispatched by the scheduler to run at a time point, and the scheduler is non-preemptive, that is, the running process returns control to the kernel only when it finishes executing or explicitly suspends itself by calling wait. Like hardware modeling languages, the SystemC scheduler supports the notion of delta-cycles [16]. A delta-cycle lasts for an infinitesimal amount of time and is used to impose a partial order of simultaneous actions which interprets zero-delay semantics. Thus, the simulation time is not advanced when the scheduler processes a delta-cycle. During a delta-cycle, the scheduler executes actions in two phases: the evaluate and the update phases.

The simulation semantics of the SystemC scheduler is presented as follows: (1) Initialize. During the initialization, each process is executed once unless it is turned off by calling dont_initialize(), or until a synchronization point (i.e., a wait) is reached. The order in which these processes are executed is unspecified; (2) Evaluate. The kernel starts a delta-cycle and runs all processes that are ready to run one at a time. In this same phase a process can be made ready to run by an event notification; (3) Update. Execute any pending calls to update() resulting from calls to request_update() in the evaluate phase. Note that a primitive channel uses request_update() to have the kernel call its update() function after the execution of processes; (4) Delta-cycle notification. The kernel enters the delta notification phase where notified events trigger their dependent processes. Note that immediate notifications may make new processes runnable during step (2). If so the kernel loops back to step (2) and starts another evaluation phase and a new delta-cycle. It does not advance simulation time; (5) Simulation-cycle notification. If there are no more runnable processes, the kernel advances simulation time to the earliest pending timed notification. All processes sensitive to this event are triggered, the kernel loops back to step (2) and starts a new delta-cycle. This process is finished when all processes have terminated or the specified simulation time is passed.

\section{B. Statistical Model Checking}

We first recall the syntax and semantics of BLTL [22], an extension of Linear Temporal Logic (LTL) with time bounds on temporal operators. A formula $\varphi$ is defined over a set of atomic propositions $A P$ as in LTL by the grammar $\varphi::=$ true $\mid$ false $|p \in A P| \varphi_{1} \wedge \varphi_{2}|\neg \varphi| \varphi_{1} U_{\leq T} \varphi_{2}$, where the time bound $T$ is an amount of time or a number of states in the execution trace. The temporal modalities $F$ (the "eventually", sometimes in the future) and $G$ (the "always", from now on forever) can be derived from the "until" $U$ as follows.

$$
F_{\leq T} \varphi=\text { true } U_{\leq T} \varphi \text { and } G_{\leq T} \varphi=\neg F_{\leq T} \neg \varphi
$$

The semantics of BLTL is defined w.r.t execution traces of a model. Let $\omega=\left(s_{0}, t_{0}\right)\left(s_{1}, t_{1}\right) \ldots\left(s_{N-1}, t_{N-1}\right), N \in \mathbb{N}$ be an execution trace, $\omega_{k}$ and $\omega^{k}$ be the prefix and suffix of $\omega$ respectively. We denote the fact that $\omega$ satisfies the BLTL formula $\varphi$ by $\omega=\varphi$. 
- $\omega^{k}=$ true and $\omega^{k} \not=$ false

- $\omega^{k} \models p, p \in A P$ iff $p \in L\left(s_{k}\right)$, where $L\left(s_{k}\right)$ is the set of atomic propositions which are true in state $s_{k}$

- $\omega^{k}=\varphi_{1} \wedge \varphi_{2}$ iff $\omega^{k} \models \varphi_{1}$ and $\omega^{k} \models \varphi_{2}$

- $\omega^{k}=\neg \varphi$ iff $\omega^{k} \not=\varphi$

- $\omega^{k}=\varphi_{1} U_{\leq T} \varphi_{2}$ iff there exists $i \in \mathbb{N}$ such that $\omega^{k+i} \models$ $\varphi_{2}, \Sigma_{0<j \leq i}\left(t_{k+j}-t_{k+j-1}\right) \leq T$, and for each $0 \leq j<$ $i, \omega^{k+j} \models \varphi_{1}$

Let $\mathcal{M}$ be the formal model of the MUV (i.e., a stochastic process) and $\varphi$ be a property expressed as a BLTL formula. The statistical model checking [15] problem consists of answering the following questions: (i) Is the probability that $\mathcal{M}$ satisfies $\varphi$ greater or equal to a threshold $\theta$ with a specific level of statistical confidence (qualitative analysis, written $\left.\mathcal{M} \models P r_{\geq \theta}(\varphi)\right)$ ? (ii) What is the probability that $\mathcal{M}$ satisfies $\varphi$ with a specific level of statistical confidence (quantitative analysis, written $\mathcal{M}=\operatorname{Pr}(\varphi)$ )? Many statistical model checker are implemented [27], [2] that have shown their advantages over other methods such as PMC on several case studies.

This is done by associating each execution trace of $\mathcal{M}$ with a discrete random Bernoulli variable $B_{i}$, in which the outcome for $B_{i}$, denoted by $b_{i}$, is 1 if the trace satisfies $\varphi$ and 0 otherwise. The predominant statistical method for verifying $\mathcal{M} \models \operatorname{Pr}_{>\theta}(\varphi)$ is based on hypothesis testing. Let $p=\operatorname{Pr}(\varphi)$, to determine whether $p \geq \theta$, we test the hypothesis $H_{0}: p \geq p_{0}=\theta+\delta$ against the alternative hypothesis $H_{1}: p \leq p_{1}=\theta-\delta$ based on the observations of $B_{i}$. The size of indifference region is defined by $p_{0}-p_{1}$. If we take acceptance of $H_{0}$ to mean acceptance of $\operatorname{Pr}_{\geq \theta}(\varphi)$ as true and acceptance of $H_{1}$ to mean rejection of $\operatorname{Pr}_{\geq \theta}(\varphi)$ as false, then we can use acceptance sampling (e.g., Younes in [26] has proposed two solutions, called single sampling plan and sequential probability ratio test) to verify $\operatorname{Pr}_{\geq \theta}(\varphi)$. An acceptance sampling test with strength $(\alpha, \beta)$ guarantees that $H_{1}$ is accepted with probability at most $\alpha$ when $H_{0}$ holds and $H_{0}$ is accepted with probability at most $\beta$ when $H_{1}$ holds, called a Type-I error and Type-II error, respectively.

To answer the quantitative question, $\mathcal{M} \models \operatorname{Pr}(\varphi)$, an alternative statistical method, based on estimation instead of hypothesis testing, has been developed. For instance, the probability estimations are based on results derived by Chernoff and Hoeffding bounds [11]. This approach uses $n$ observations $b_{1}, \ldots, b_{n}$ to compute an approximation of $p: \tilde{p}=\frac{1}{n} \sum_{i=1}^{n} b_{i}$. The approximation satisifies that $\operatorname{Pr}[|\tilde{p}-p|<\delta] \geq 1-\alpha$. Based on the theorem of Hoeffding, the number of observations which is determined from the absolute error $\delta$ and the confidence $1-\alpha$ is $n=\left\lceil\frac{1}{2 \delta^{2}} \log \frac{2}{\alpha}\right\rceil$.

Although SMC can only provide approximate results with a user-specified level of statistical confidence, it is compensated for by its better scalability and resource consumption. Since the models to be analyzed are often approximately known, an approximate result in the analysis of desired properties within specific bounds is quite acceptable. SMC has recently been applied in a wide range of research areas including software engineering (e.g., verification of critical embedded systems) [9], system biology, or medical area [12].

\section{A RUNNING EXAMPLE}

We will use a simple case study with a FIFO channel as a running example. This example illustrates how designers can create hierarchical channels that encapsulate both design structure and communication protocols. In the design, once a nanosecond the producer will write one character to the FIFO with probability $p_{1}$, while the consumer will read one character from the FIFO with probability $p_{2}$. The FIFO which is derived from $s c$ channel encapsulates the communication protocol between the producer and the consumer.

The FIFO channel is designed to ensure that all data is reliably delivered despite the varying rates of production and consumption. The channel uses an event notification hanshake protocol for both the input and output. It uses a circular buffer implemented within a static array to store and retrieve the items within the FIFO. We assume that the sizes of the messages and the FIFO buffer are fixed. Hence, it is obvious that the time required to transfer completely a message, or message latency, depends on the production and consumption rates, the FIFO buffer size, the message size, and the probabilities of successful writing and reading. The full implementation of the example can be obtained at the $\mathrm{url}^{2}$, in which the probabilities of writing and reading are implemented with the Bernoulli distributions with probabilities $p_{1}$ and $p_{2}$ respectively from GNU Scientific Library (GSL) [7].

The quantitative analysis under consideration is: "What is the probability that messages are transfered completely within $T_{1}$ nanoseconds during $T$ nanoseconds of operation?". This kind of analysis can, thus, be conducted in the early design steps. To formulate the underlying property more precisely, we have to take into account the agreement protocol between the producer and consumer, i.e., the simple protocol can be every message has special starting delimiter with the character ' $\&$ ' and ending delimiter with the character '@'. Thus, the property can be translated in BLTL as follows:

$$
\varphi=G_{\leq T}\left(\left(c \_r e a d='{ }^{\prime}\right) \rightarrow F_{\leq T_{1}}\left(c \_r e a d={ }^{\prime} @^{\prime}\right)\right)
$$

where $c$ read is the character read in the FIFO by the consumer. The input providing to the SMC checker is $\operatorname{Pr}(\varphi)$. This property is expressed in terms of the characters read in the FIFO by the consumer, but the communication protocol between the producer and the consumer is abstracted at a very high level. It is an illustration of the types of properties that can be checked on TLM specifications. The verification of such a property at the transaction level can be connected to its counterpart at register-transfer level (RTL) in order to check the correctness of RTL implementations.

\section{SMC FOR SYSTEMC MODELS}

\section{A. SystemC Model State}

Temporal logic formulas are interpreted over execution traces and traditionally a trace has been defined as a sequence

\footnotetext{
${ }^{2}$ https://project.inria.fr/plasma-lab/files/2015/09/producer_consumer.tar_.gz
} 
of states in the execution of a model. Therefore before we can define an execution trace we need a precise definition of the state of a SystemC model simulation. We are inspired by the definition of system state in [23], which consists of the state of the simulation kernel and the state of the SystemC model. We consider the external libraries as black boxes, meaning that their states are not exposed.

The state of the kernel contains the information about the current phase of the simulation (i.e., delta-cycle notification, simulation-cycle simulation) and the SystemC events notified during the execution of the model. The state of the SystemC model is the full state of the $\mathrm{C}++$ code of all modules in the model, which includes the values of the module attributes, the location of the program counter (i.e., a particular statement is reached during the execution of the model, the function calls), the call stack including the function execution, function parameters and return values, and the status of the module processes (i.e., suspended, runnable). We use $V=\left\{v_{0}, \ldots, v_{n-1}\right\}$ to denote the finite set of variables of primitive type (e.g, usual scalar or enumerated type in $\mathrm{C} / \mathrm{C}++$ ) whose value domain $\mathbb{D}_{X}$ represents the states of a SystemC model.

We consider here some examples about states of the simulation kernel and the SystemC model. Assume that a SystemC model has an event named $e$, then the model state can contain information such as the kernel is at the end of simulation-cycle notification phase and the event $e$ is notified. Consider the running example again, a state can consist of the information about the characters received by the consumer, represented by the variable c_read. It also contains the information about the location of the program counter right before and after a call of the function send() in the module Producer that are represented by two Boolean variables send_start and send_done, respectively, meaning that they hold the value true immediately before and after a call of the function send(). Another example, we consider a module that consists several statements at different locations in the source code, in which these statements contain the division operator " " followed by zero or more spaces and the variable " $a$ " (e.g., the statement $y$ $=(x+1) / a)$. Then, a Boolean variable which holds the value true right before the execution of all such statements can be used as a part of the states.

We have discussed so far the state of a SystemC model execution. It remains to discuss how the semantics of the temporal operators is interpreted over the states in an execution. That means how the states are sampled in order to make the transition from one state to another state. The following definition gives the concept of temporal resolution, in which the states are evaluated only at instances in which the temporal resolution holds. It allows the user to set granularity of time.

Definition 1 (Temporal resolution): A temporal resolution $\mathcal{T}_{r}$ is a finite set of Boolean expressions defined over $V$ which specifies when the set of variables $V$ is evaluated.

Temporal resolution can be used to define a more fine-grained model of time than a coarse-grained one provided by a cyclebased simulation. We call the expressions in $\mathcal{T}_{r}$ temporal events. Whenever a temporal event is satisfied or the temporal event occurs, $V$ is sampled. For example, in the producer and consumer model, assume that we want the satisfaction of the underlying BLTL $\varphi$ to be checked whenever at the end of simulation-cycle notification or immediately after the event write_event is notified during a run of the model. Hence, we can define a temporal resolution as the following set $\mathcal{T}_{r}=$ $\{$ end_sc,we_notified $\}$, where end_sc and we_notified are Boolean expressions that have the value true whenever the kernel phase is at the end of the simulation-cycle notification and the event write_event notified, respectively.

We denote the set of occurrences of temporal events from $\mathcal{T}_{r}$ along an execution of a SystemC model by $\mathcal{T}_{r}^{s}$, called a temporal resolution set. The value of a variable $v \in V$ at an event occurrence $e_{c} \in \mathcal{T}_{r}^{s}$ is defined by a mapping $\xi_{\text {val }}^{v}: \mathcal{T}_{r}^{s} \rightarrow$ $\mathbb{D}_{v}$, where $\mathbb{D}_{v}$ is the value domain of $v$. Hence, the state of the SystemC model at $e_{c}$ is defined by a tuple $\left(\xi_{\text {val }}^{v_{0}}, \ldots, \xi_{\text {val }}^{v_{n-1}}\right)$.

A mapping $\xi_{t}: \mathcal{T}_{r}^{s} \rightarrow \mathcal{T}$ is called a time event that identifies the simulation time at each occurrence of an event from the temporal resolution. Hence, the set of time points, called time tag, which corresponds to a temporal resolution set $\mathcal{T}_{r}^{s}=$ $\left\{e_{c_{0}}, \ldots, e_{c_{N-1}}\right\}, N \in \mathbb{N}$, is given as follows.

Definition 2 (Time tag): Given a temporal resolution set $\mathcal{T}_{r}^{s}$, the time tag $\mathcal{T}$ corresponding to $\mathcal{T}_{r}^{s}$ is a finite or infinite set of non-negative reals $\left\{t_{0}, t_{1}, \ldots, t_{N-1}\right\}$, where $t_{i+1}-t_{i}=$ $\delta t_{i} \in \mathbb{R}_{\geq 0}, t_{i}=\xi_{t}\left(e_{c_{i}}\right)$.

\section{B. Model and Execution Trace}

A SystemC model can be viewed as a hierarchical network of parallel communicating processes. Hence, the execution of a SystemC model is an alternation of the control between the model's processes, the external libraries and the kernel process. The execution of the processes is supervised by the kernel process to concurrently update new values for the signals and variables w.r.t the cycle-based simulation. For example, given a set of runnable processes in a simulation-cycle, the kernel chooses one of them to execute first in a non-deterministic manner as described in the prior section.

Let $V$ be the set of variables whose values represent the states of a SystemC model. The values of variables in $V$ are determined by a given probability distribution (i.e., production from all probability distributions used in the model). Given a temporal resolution $\mathcal{T}_{r}$ and its corresponding temporal resolution set along an execution of the model $\mathcal{T}_{r}^{s}=\left\{e_{c_{0}}, \ldots, e_{c_{N-1}}\right\}, N \in \mathbb{N}$, the evaluation of $V$ at the event occurrence $e_{c_{i}}$ is defined by the tuple $\left(\xi_{v a l}^{v_{0}}, \ldots, \xi_{v a l}^{v_{n-1}}\right)$, or a state of the model at $e_{c_{i}}$, denoted by $V\left(e_{c_{i}}\right)=\left(V\left(e_{c_{i}}\right)\left(v_{0}\right), V\left(e_{c_{i}}\right)\left(v_{1}\right), \ldots, V\left(e_{c_{i}}\right)\left(v_{n-1}\right)\right)$, where $V\left(e_{c_{i}}\right)\left(v_{k}\right)=\xi_{\text {val }}^{v_{k}}\left(e_{c_{i}}\right)$ with $k=0, \ldots, n-1$ is the value of the variable $v_{k}$ at $e_{c_{i}}$. We denote the set of all possible evaluations by $V_{\mathcal{T}_{r}^{s}} \subseteq \mathbb{D}_{V}$, called the state space of the random variables in $V$. State changes are observed only at the moments of event occurrences. Hence, the operational semantics of a SystemC model is represented by a stochastic process $\left\{\left(V\left(e_{c_{i}}\right), \xi_{t}\left(e_{c_{i}}\right)\right), e_{c_{i}} \in \mathcal{T}_{r}^{s}\right\}_{i \in \mathbb{N}}$, taking values in $V_{\mathcal{T}_{r}^{s}} \times \mathbb{R}_{\geq 0}$ and indexed by the parameter $e_{c_{i}}$, which are event occurrences in the temporal resolution set $\mathcal{T}_{r}^{s}$. An execution 
trace is a realization of the stochastic process is given as follows.

Definition 3 (Execution trace): An execution trace of a SystemC model corresponding to a temporal resolution set $\mathcal{T}_{r}^{s}=\left\{e_{c_{0}}, \ldots, e_{c_{N-1}}\right\}, N \in \mathbb{N}$ is a sequence of states and event occurrence times, denoted by $\omega=$ $\left(s_{0}, t_{0}\right) \ldots\left(s_{N-1}, t_{N-1}\right)$, such that for each $i \in 0, \ldots, N-1$, $s_{i}=V\left(e_{c_{i}}\right)$ and $t_{i}=\xi_{t}\left(e_{c_{i}}\right)$.

$N$ is the length (finite or infinite) of the execution, also denoted by $|\omega|$. Let $V^{\prime} \subseteq V$, the projection of $\omega$ on $V^{\prime}$, denoted by $\omega \downarrow_{V^{\prime}}$, is an execution trace such that $\left|\omega \downarrow_{V^{\prime}}\right|=|\omega|$ and $\forall v \in V^{\prime}, \forall e_{c} \in \mathcal{T}_{r}^{s}, V^{\prime}\left(e_{c}\right)(v)=V\left(e_{c}\right)(v)$.

\section{Expressing Properties}

Our approach allows users to refer to a rich set of atomic propositions $A P$ which is defined over the set of variables $V$ as previously mentioned. These propositions abstract the states of a SystemC model and evaluate to either true or false in such a state. The implementation provides a mechanism that allows users to declare $V$ in order to define the set of propositions $A P$ without requiring users to write the monitoring code or to write aspect-oriented programming advices manually.

Users declare these variables via a high-level language in a configuration file as the input of our tool. For instance, referring to the producer and consumer model, the declaration location send_start "\%Producer::send()":call declares a Boolean variable send_start that holds the value true immediately before the execution of the model reaches a call site of the function send() in the module Producer. The characters received by the consumer which is represented by the variable c_read can be declared as attribute pnt_con $\rightarrow$ c_int c_read, where pnt_con is a pointer to the Consumer object and $c_{-}$int is an attribute of the Consumer module representing the received character. The detailed syntax can be found in the tool manual ${ }^{3}$.

$A P$ are predicates defined over the set of variables $V$. Using these predicates, users can define temporal properties related to the states of the kernel and the SystemC model. Recall the considered property of the running example, the predicates which are defined over the variable $c \_r e a d$ are $c \_r e a d={ }^{\prime} \&^{\prime}$ and c_read $={ }^{\prime} @^{\prime}$. Another example, assume that we want to answer the following question: "Over a period of $T$ time units, is the probability that the number of elements in the FIFO buffer in between $n_{1}$ and $n_{2}$ is greater or equal to $\theta$ with the confidence $\alpha$ ?"'. The predicates need to be defined in order to construct the underlying BLTL formula are $n_{1} \leq n_{\text {elements }}$ and $n_{\text {elements }} \leq n_{2}$, where $n_{\text {elements }}$ is an integer variable that represents the current number of elements in the FIFO buffer (it captures the value of the num_elements attribute in the Fifo module). Then, the property can be translated in BLTL with the operator "always" as follows. The input which is given to the checker is $\operatorname{Pr}_{\geq \theta}(\varphi)$ along with the confidence $\alpha$.

$$
\varphi=G_{\leq T}\left(\left(n_{1} \leq n_{\text {elements }}\right) \&\left(n_{\text {elements }} \leq n_{2}\right)\right)
$$

\footnotetext{
${ }^{3}$ https://project.inria.fr/plasma-lab/documentation/tutorial/mag_manual/
}

\section{IMPLEMENTATION}

\section{A. MAG and SystemC Plugin}

Fig. 1 shows our SMC-based verification tool implementation that contains two main components: a monitor and aspectadvice generator (MAG) and a statistical model checker (SystemC Plugin). In principle, the full state can be observed during the simulation of the model. In practice, however, users define a set of variables of interest, according to the properties that the users want to verify, called observed variables, and only these variables appear in the states of an execution trace. Given a SystemC model, we use $V_{o b s} \subseteq V$ to denote

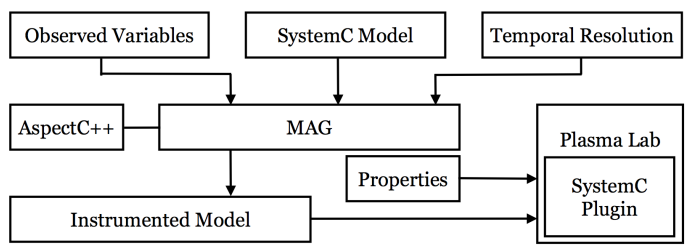

Fig. 1: The framework's flow

the set of observed variables, to expose the states of the SystemC model. Then, the observed execution traces of the model are the projections of the execution traces on $V_{o b s}$, meaning that for every execution trace $\omega$, the corresponding observed execution trace is $\omega \downarrow_{V_{\text {obs }}}$. In the following, when we mention execution traces, we mean observed execution traces. The implementation of MAG allows users to define a set of observed variables that is used with a temporal resolution to generate a monitor. The implementation based on the techniques in [23], in which a monitor and a file containing aspects are generated in order to automatically instrument the SystemC model with the help of AspectC++ [5] and establish a communication between the generated monitor and the instrumented model. The monitor evaluates the set of observed variables at every time point at which an event of the temporal resolution occurs during the SystemC model simulation to produce a new state. For example, the variable c_read which observes the character received by the consumer (the private attribute $c \_$int in the module Consumer) at the end of simulation-cycle notification, is implemented by generating a monitor and instrumenting the module Consumer to establish a communication between them as follows. The module Consumer is instrumented with AspectC++, in this case, such that the monitor is its friend class, so the monitor can access the private attributes of Consumer. The monitor defines a callback function being called immediately at the end of simulationcycle notification, and a pointer pointing to an instance of Consumer. The execution of the callback function consists of getting the current value of the received character by the consumer, assigning this character to $c \_r e a d$, and executing the monitor for one step (i.e., creating a new state and reporting it to the Plasma plugin). If temporal resolutions involving kernel simulation phases or event notifications are defined, the calling mechanism of the callback function is realized 
by modifiying the kernel (i.e., at the end of simulation-cycle segment code, a call to the callback function is added).

The statistical model checker is implemented as a plugin of Plasma Lab [2] that establishes a communication, in which the generated monitor transmits execution traces of the MUV. In the current version, the communication is done via the standard input and output. When a new state is requested, the monitor reports the current state (the values of variables in $V_{o b s}$ ) to the plugin. The length of traces depends on the satisfaction of the formula to be verified, which is finite because the temporal operators are bounded. Similarly, the required number of execution traces depends on the hypothesis testing algorithms in use (e.g., sequential hypothesis testing or 2-sided Chernoff bound). The full implementation can be downloaded on the website of Plasma $\mathrm{Lab}^{4}$.

\section{B. Running Verification}

Running the verification tool consists of two steps as follows. First, users define a set of observed variables and a temporal resolution in a configuration file, as well as other necessary information as an input for MAG to generate a monitor and an aspect-advices file. AspectC++ is then used to instrument automatically the model. The instrumented model and the generated monitor are compiled and linked together with the SystemC kernel into an executable model in order to make a set of execution traces. Referring to the running example, users will define the set of observed variables $V_{o b s}=$ $\left\{c \_r e a d, n_{\text {elements }}, e n d \_s c\right\}$, where $c \_r e a d$ is the character read in the FIFO, $n_{\text {elements }}$ is the number of characters in the FIFO buffer, and end_sc is true whenever the kernel phase is at the end of the simulation-cycle notification phase. The temporal resolution will be defined as $\mathcal{T}_{r}=\left\{e n d \_s c\right\}$, meaning that a new state in execution traces is produced whenever the simulation kernel is at the end of simulation-cycle notification phase or every one nanosecond in the example since the time unit is one nanosecond. The full configuration file is included in the source code of the example.

In the second step, the plugin is used to verify the properties of interest. The satisfaction checking of the properties is brought out based on the set of execution traces obtained by executing the instrumented SystemC model and can be done by several hypothesis testing algorithms provided by Plasma Lab.

\section{CASe Studies}

We report the experimental results for the running example and also demonstrate the use of our verification tool to analyze the dependability of a large embedded control system. The number of components in this system makes numerical approaches such as PMC infeasible. In both case studies, we used the 2-sided Chernoff bound algorithm with the absolute error $\delta=0.02$ and the confidence $1-\alpha=0.98$. The experiments were run on a machine with an Intel Core i7 2.67 GHz processor and 4GB RAM under the Linux OS with

\footnotetext{
${ }^{4}$ https://project.inria.fr/plasma-lab/download/plugins/
}

SystemC 2.3.0, in which the checking of the properties in the running example took from less than one minute to several minutes. The analysis of the embedded and control system case study takes almost 2 hours, in which 90 properties were verified.

\section{A. Producer and Consumer}

Let us go back to the running example in Section III, recall that we want to compute the probability that the following property $\varphi$ satisfies every 1 nanosecond, with the absolute error 0.02 and the level of confidence 0.98 . In this verification, both the FIFO buffer size and message size are 10 characters including the starting and ending delimiters, and the production and consumption rates are 1 nanosecond. First, we check this property with the various values of $p_{1}$ and $p_{2}$. The results are given in Table I with $T=5000$ and $T_{1}=25$ nanoseconds. It is trivial that the probability that the message latency is smaller than $T_{1}$ time increases when $p_{1}$ and $p_{2}$ increase. That means that, in general, the latency is shorter when the either the probability that the producer successfully writes to the FIFO increases, or the probability that the consumer successfully reads from the FIFO increases. Second, we compute the probability that a message is sent

\begin{tabular}{c|ccc}
$p_{1} \backslash p_{2}$ & 0.3 & 0.6 & 0.9 \\
\hline 0.6 & 0 & 0.0194 & 0.0720 \\
0.9 & 0 & 0.0835 & 1
\end{tabular}

TABLE I: The probability that the message latency is smaller than 25 in the first 5000 nanoseconds of operation

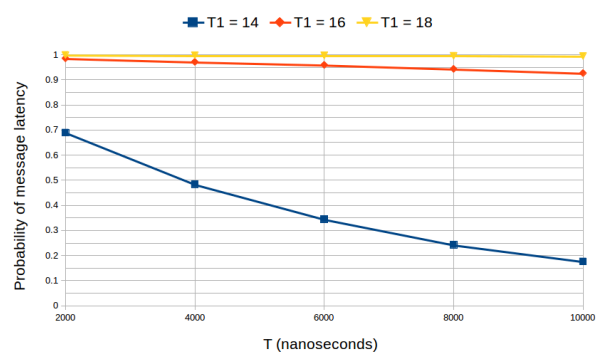

Fig. 2: The probability that the message latency is smaller than $T_{1}$ in the first $T$ nanoseconds of operation

completely (or the message latency) from the producer to the consumer within $T_{1}$ time over a period of $T$ time of operation, in which the probabilities $p_{1}$ and $p_{2}$ are fixed at 0.9 . Fig. 2 shows this probability with different values of $T_{1}$ over $T=10000$ nanoseconds. It is observed that the message latency is almost smaller than 18 nanoseconds.

\section{B. An Embedded Control System}

This case study is closely based on the one presented in [20], [14] but contains much more components. The system consists of an input processor (I) connected to 50 groups of 
3 sensors, an output processor $(O)$, connected to 30 groups of 2 actuators, and a main processor $(M)$, that communicates with $I$ and $O$ through a bus. At every cycle, 1 minute, the main processor polls data from the input processor that reads and processes data from the sensor groups. Based on this data, the main processor constructs commands to be passed to the output processor for controlling the actuator groups.

The reliability of the system is affected by the failures of the sensors, actuators, and processors. The probability of bus failure is negligible, hence we do not consider it. The sensors and actuators are used in $37-$ of -50 and $27-$ of -30 modular redundancies, respectively. That means if at least 37 sensor groups are functional (a sensor group is functional if at least 2 of the 3 sensors are functional), the system has enough information to function properly. Otherwise, the main processor is reported to shut the system down. In the same way, the system requires at least 27 functional actuator groups to function properly (a actuator group is functional if at least 1 of the 2 actuators is functional). Transient and permanent faults can occur in processors $I$ or $O$ and prevent the main processor $(M)$ to read data from $I$ or send commands to $O$. In that case, $M$ skips the current cycle. If the number of continuously skipped cycles exceeds the limit $K$, the processor $M$ shuts the system down. When a transient fault occurs in a processor, rebooting the processor repairs the fault. Lastly, if the main processor fails, the system is automatically shut down. The mean times to failure for the sensors, the actuators, and the processors are 1 month, 2 months and 1 year, respectively. The mean time to transient failure is 1 day and $\mathrm{I} / \mathrm{O}$ processors take 30 seconds, 1 time unit, to reboot.

The reliability of the system is modeled as a CTMC [18], [25] that is realized in SystemC, in which a sensor group has 4 states $(0,1,2,3$, the number of working sensors), 3 states $(0,1,2$, the number of working actuators) for an actuator group, 2 states for the main processor (0: failure, 1: functional), and 3 states for I/O processors ( 0 : failure, 1: transient failure, 2: functional). A state of the CTMC is represented as a tuple of the component's states, and the mean times to failure define the delay before which a transition between states is enabled. The delay is sampled from a negative exponential distribution with parameter equal to the corresponding mean time to failure. Hence, the model has about $2^{155}$ states comparing to the model in [14] with about $2^{10}$ states, that makes the PMC technique is unfeasible. That means the state explosion likely occurs, even with some abstraction, i.e., symbolic model checking is applied. The full implementation of the SystemC code and experiments of this case study can be obtained at the website of our tool ${ }^{5}$. We define four types of failures: failure ${ }_{1}$ is the failure of the sensors, failure f $_{2}$ is the failure of the actuators, failure f $_{3}$ is the failure of the $\mathrm{I} / \mathrm{O}$ processors and failure $_{4}$ is the failure

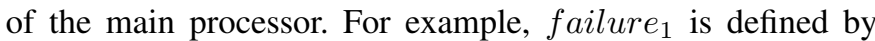
number_sensors $<37) \wedge($ proci_status $=2)$. It specifies that the number of working sensor groups has decreased

\footnotetext{
${ }^{5}$ https://project.inria.fr/plasma-lab/embedded-control-system/
}

below 37 and the input processor is functional, so that it can report the failure to the main processor. We define failure $_{2}$, failure $_{3}$, and failure $_{4}$ in a similar way.

In our analysis which is based on the one in [14] with $K=4$, in which the properties are checked every 1 time unit. First, we try to determine which kind of component is more likely to cause the failure of the system, meaning that we determine the probability that a failure related to a given component occurs before any other failures. The atomic proposition shutdown $=\bigvee_{i=1}^{4}$ failure $_{i}$ indicates that the system has shut down because one of the failures has occurred, and the BLTL formula $\neg$ shutdown $U_{\leq T}$ failure $_{i}$ states that the failure $i$ occurs within $T$ time units and no other failures have occurred before the failure $i$ occurs. Fig. 3 shows the probability that each kind of failure occurs first over a period of 30 days of operation. It is obvious that the sensors are likelier to cause a system shutdown. At $T=20$ days, it seems that we reached a stationary distribution indicating for each kind of component the probability that it is responsible for the failure of the system.

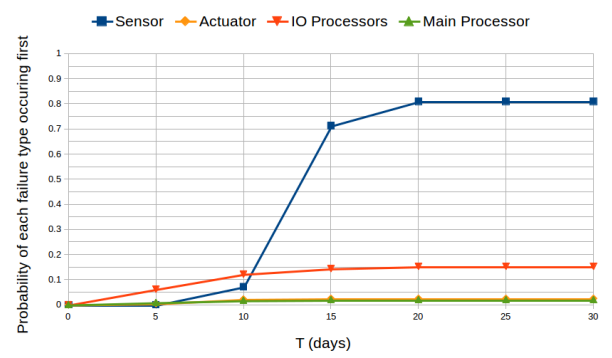

Fig. 3: The probability that each of the 4 failure types is the cause of system shutdown in the first $T$ time of operation

For the second part of our analysis, we divide the states of system into three classes: "up", where every component is functional, "danger", where a failure has occurred but the system has not yet shut down (e.g., the I/O processors have just had a transient failure but they have rebooted in time), and "shutdown", where the system has shut down [14]. We aim to compute the expected time spent in each class of states by the system over a period of $T$ time units. To this end, we add in the model, for each class of state $c$, a random variable reward_c that measures the time spent in the class $c$. In our tool, the formula $X_{\leq T}$ reward_c returns the mean value of reward_c after $T$ time of execution. The results are plotted in Fig. 4. From $T=20$ days, it seems that the amounts of time spent in the "up" and "danger" states are converged at $10^{1.063}=11.57$ days and $10^{-1.967}=0.01$ days, respectively.

\section{RELATED WORK AND CONCLUSION}

There has been a lot of work on the formalization of SystemC [8], [17]. The goal is to extract a formal model from a SystemC program, so that tools like model-checkers can be applied. However, all these formalizations consider semantics 


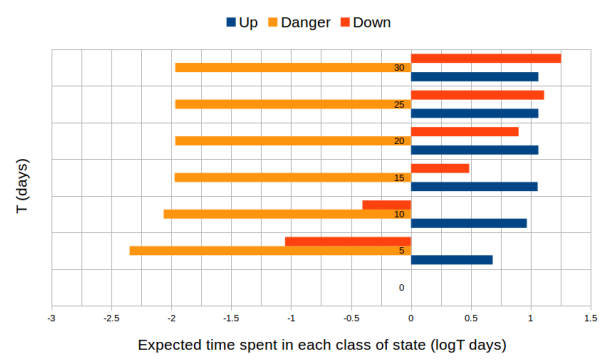

Fig. 4: The expected amount of time spent in each of the states: "up", "danger" and "shutdown"

of SystemC and its simulator in some form of global model, and they also suffer from the state space explosion when dealing with industrial and large systems.

In [29], Zuliani et al. extended the standard SMC algorithm for verifying Stateflow/Simulink models of a fuel control system featuring fault-tolerance and hybrid behavior, in which properties under verification are expressed in BLTL. The extension is based on Bayesian interval estimation and Bayesian sequential hypothesis testing. This technique is scalable for larger Stateflow/Simulink models because verification is fast in most cases and the Bayesian estimation is orders of magnitudes faster than previous estimation-based model checking algorithms.

Tabakov et al. [23] proposed a framework for monitoring temporal SystemC properties. This framework allows users to express the properties to verify by fully exposing the semantics of the simulator as well as the user-code. They extend LTL by providing some extra primitives for stating the atomic propositions and let users define a much finer temporal resolution. Their implementation consists of a modified simulation kernel, and a tool to automatically generate the monitors and aspect advices for instrumenting SystemC programs automatically.

This paper presents the first attempt to verify non-trivial temporal properties of a SystemC model with statistical model checking techniques. The framework contains two main components: a generator that automatically generates a monitor and instruments the MUV based on the properties to be verified, and a statistical model checker implementing a set of hypothesis testing algorithms. In comparison to the probabilistic model checking, our approach allows users to handle large industrial systems, expose a rich set of user-code primitives in form of atomic propositions in BLTL, and work directly with SystemC models. For instance, our verification framework is used to analyze the dependability of large industrial computerbased control systems as shown in the case study.

Currently, we consider an external library as a "black box", meaning that we do not consider the states of external libraries. Thus, arguments passed to a function in an external library cannot be monitored. For future work, we would like to allow users to monitor the states of the external libraries. We also plan to apply statistical model checking to verify temporal properties of SystemC-AMS (Analog/Mixed-Signal).

\section{REFERENCES}

[1] Accellera. http://www.accellera.org/downloads/standards/systemc.

[2] B. Boyer, K. Corre, A. Legay, and S. Sedwards. Plasma lab: A flexible, distributable statistical model checking library. In QEST'13, 2013.

[3] H. Chang, L. Cooke, M. Hunt, G. Martin, A. McNelly, and L. Todd. Surviving the soc revolution: A guide to platform-based design. In Norwell, USA, 1999.

[4] F. Ciesinski and M. Grober. On probabilistic computation tree logic. In Validation of Stochastic Systems, 2004.

[5] A. Gal, W. Schroder-Preikschat, and O. Spinczyk. Aspectc++: Language proposal and prototype implementation. In OOPSLA'01, 2001.

[6] T. Grotker, S. Liao, G. Martin, and S. Swan. System Design with SystemC. Kluwer Academic Publishers, Norwell, USA, 2002.

[7] GSL. http://www.gnu.org/software/gsl/.

[8] P. Herber, J. Fellmuth, and S. Glesner. Model checking systemc designs using timed automata. In CODES/ISSS'08, 2008.

[9] H. Hermanns, B. Watcher, and L. Zhang. Probabilistic cegar. In CAV'08. LNCS, Springer, 2008.

[10] A. Hinton, M. Kwiatkowska, G. Norman, and D. Parker. Prism: A tool for automatic verification of probabilistic systems. In TACAS'06. LNCS, Springer, 2006.

[11] W. Hoeffding. Probability inequalities for sums of bounded random variables. In American Statistical Association, 1963.

[12] S. Jha, E. Clarke, C. Langmead, A. Legay, A. Platzer, and P. Zuliani. A bayesian approach to model checking biological systems. In $C M S B^{\prime} 09$. LNCS, Springer, 2009.

[13] J. Katoen, E. Hahn, H. Hermanns, D. Jansen, and I. Zapreev. The ins and outs of the probabilistic model checker mrmc. In QEST'09, 2009.

[14] M. Kwiatkowska, G. Norman, and D. Parker. Controller dependability analysis by probabilistic model checking. In Control Engineering Practice. Elsevier, 2007.

[15] A. Legay, B. Delahaye, and S. Bensalem. Statistical model checking: An overview. In $R V^{\prime} 10,2010$.

[16] R. Lipsett, C. Schaefer, and C. Ussery. VHDL: Hardware description and design. Kluwer Academic Publishers, 1993.

[17] F. Maraninchi, M. Moy, C. Helmstetter, J. Cornet, C. Traulsen, and L. Maillet-Contoz. Systemc/tlm semantics for heterogeneous socs validation. In NEWCAS/TAISA'08, 2008.

[18] M. A. Marsan and M. Gerla. Markov models for multiple bus multiprocessor systems. In IEEE Transactions on Computer, 1982.

[19] W. Mueller, J. Ruf, D. Hoffmann, J. Gerlach, T. Kropf, and W. Rosenstiehl. The simulation semantics of systemc. In DATE 2001, 2001.

[20] J. Muppala, G. Ciardo, and K. Trivedi. Stochastic reward nets for reliability prediction. In CRMS'94, 1994.

[21] J. Rutten, M. Kwiatkowska, G. Norman, and D. Parker. Mathematical techniques for analyzing concurrent and probabilistic systems. In CRM Monograph Series, 2004.

[22] K. Sen, M. Viswanathan, and G. Agha. On statistical model checking of stochastic systems. In CAV'05, 2004.

[23] D. Tabakov and M. Vardi. Monitoring temporal systemc properties. In Formal Methods and Models for Codesign, 2010.

[24] D. Thomas and P. Moorby. The verilog hardware description language. In Springer. ISBN 0-3878-4930-0, 2008.

[25] K. S. Trivedi. Probability and statistics with reliability, queueing, and computer science applications. In Englewood Cliffs, 1982.

[26] H. Younes. Verification and planning for stochastic processes with asynchronous events. In PhD Thesis, Carnegie Mellon, 2005.

[27] H. Younes. Ymer: A statistical model checker. In CAV'05, 2005.

[28] H. Younes, M. Kwiatkowska, G. Norman, and D. Parker. Numerical vs statistical probabilistic model checking. In STTT'06, 2006.

[29] P. Zuliani, A. Platzer, and E. M.Clarke. Bayesian statistical model checking with application to simulink/stateflow verification. In Formal Methods in System Design, 2013. 